\title{
Calculation of Some Integrals Arising in Heat Transfer in Geothermics
}

\author{
J. L. G. Santander, ${ }^{1}$ P. Castañeda Porras, $_{r}{ }^{2}$ J. M. Isidro, ${ }^{3}$ \\ and P. Fernández de Córdoba ${ }^{3}$ \\ ${ }^{1}$ Departamento de Ciencias Experimentales y Matemáticas, Universidad Católica de Valencia, \\ 46002 Valencia, Spain \\ ${ }^{2}$ Departamento de Matemáticas, Universidad de Pinar del Río, 20200 Pinar del Río, Cuba \\ ${ }^{3}$ Instituto Universitario de Matemática Pura y Aplicada, Universidad Politécnica de Valencia, \\ 46022 Valencia, Spain
}

Correspondence should be addressed to J. L. G. Santander, martinez.gonzalez@ucv.es

Received 28 September 2010; Accepted 29 November 2010

Academic Editor: Miguel Cerrolaza

Copyright (C) 2010 J. L. G. Santander et al. This is an open access article distributed under the Creative Commons Attribution License, which permits unrestricted use, distribution, and reproduction in any medium, provided the original work is properly cited.

We calculate some integrals involved in the temperature field evaluation of the ground, heated by a borehole heat exchanger. This calculation allows a faster computation of that component of the temperature field which involves the periodic oscillation of the ambient temperature or the ambient heat flux.

\section{Introduction}

Ground-coupled heat pumps (GCHPs) are an attractive choice of system for heating and cooling buildings [1]. By comparison with standard technologies, these pumps offer competitive levels of comfort, reduced noise levels, lower greenhouse gas emissions, and reasonable environmental safety. Furthermore, their electrical consumption and maintenance requirements are lower than those required by conventional systems and, consequently, have lower annual operating cost [2].

A GCHP exchanges heat with the ground through a buried U tube loop. Since this exchange strongly depends on the thermal properties of the ground, it is very important to have a knowledge of these properties when designing GCHP air-conditioning systems. Methods to estimate them include literature searches, conducting laboratory experiments on soil/rock samples, and/or performing field tests. The in situ tests are based on studying the thermal response of the borehole heat exchanger to a constant heat injection or extraction. The outputs of the thermal response test are the inlet and outlet temperature of the heat-carrier 
fluid as a function of time. From these experimental data, and with an appropriate model describing the heat transfer between the fluid and the ground, the thermal conductivity of the surroundings is inferred.

The results presented in this study are based on a model of the underground heat transfer due to a borehole heat exchanger assuming that it works as a line heat source of finite depth inside the ground. We assume as well a constant power of length unit for the heat source considered and a periodic oscillation of the ambient temperature or heat flux on the ground surface.

This paper is organized as follows. Section 2 describes the equations that model the heat transfer inside the ground due to a borehole heat exchanger. Section 3 solves the integrals given in literature for periodic oscillations of the ambient temperature or heat flux, on the ground surface. Section 4 shows that our results agree with the literature in some particular cases, such as the solution on the ground surface and the solution of the quasistationary regime.

\section{The Equations}

Let us consider the heat equation in cylindrical coordinates, in which $T(r, z, t)$ is the temperature field,

$$
\frac{\partial T}{\partial t}-k \nabla^{2} T=\frac{Q_{z}}{C} \delta(r)[H(z)-H(z-L)]
$$

On the one hand, the thermal parameters of (2.1) are the ground thermal diffusivity $k\left(m^{2} s^{-1}\right)$, the heat flow per length unit $Q_{z}\left(W^{-1}\right)$, and the volumetric heat capacity of ground $\mathrm{C}\left(\mathrm{Jm}^{-3} \mathrm{~K}^{-1}\right)$. On the other hand, the geometry of the heat source, depicted in Figure 1, has been taken into account introducing the Heaviside function $H$ for its finite length $L$ and the Dirac delta distribution $\delta$ for its infinitely thin width. Equation (2.1) is subject to to the initial condition

$$
T(r, z, 0)=T_{0}+k_{\text {geo }} z, \quad z \geq 0,
$$

where $T_{0}$ is the undisturbed ground temperature and $k_{\text {geo }}\left(\mathrm{Km}^{-1}\right)$ is the geothermal gradient. We may consider that (2.1) is subject to a Dirichlet boundary condition, which represents a time-dependent temperature on the surface $z=0$,

$$
T(r, 0, t)=\psi(t), \quad t \geq 0,
$$

or to a Neumann boundary condition, which represents a time-dependent heat flux on the surface $z=0$,

$$
-\lambda \partial_{z} T(r, 0, t)=\psi(t), \quad t \geq 0
$$




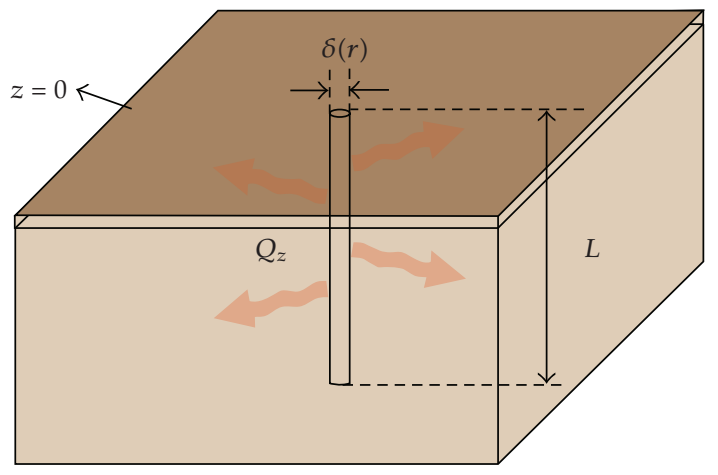

Figure 1: Borehole heat exchanger model.

where $\lambda\left(W m^{-1} K^{-1}\right)$ is the ground thermal conductivity. We split the Cauchy problem posed in (2.1), (2.2), and (2.3) or (2.4) in three terms [3], so that

$$
T(r, z, t)=v_{d}(r, z, t)+v_{0}(z, t)+v_{s}(z, t)
$$

and where the functions $v_{0}, v_{s}$ and $v_{d}$ satisfy the Cauchy problems presented below.

\subsection{The Problem for $v_{d}$}

The partial differential equation that $v_{d}(r, z, t)$ satisfies is

$$
\frac{\partial v_{d}}{\partial t}-k \nabla^{2} v_{d}=\frac{Q_{z}}{C} \delta(r)[H(z)-H(z-L)]
$$

subject to a homogeneous initial condition,

$$
v_{d}(r, z, 0)=0, \quad z \geq 0,
$$

and to a homogeneous Dirichlet boundary condition,

$$
v_{d}(r, 0, t)=0, \quad t \geq 0,
$$

or to a Neumann boundary condition, homogenous as well,

$$
\partial_{z} v_{d}(r, 0, t)=0, \quad t \geq 0 .
$$


The solution to (2.6), (2.7), and (2.8) is [4]

$$
\begin{aligned}
v_{d}(r, z, t)= & \frac{Q_{z}}{8 \pi \lambda} \int_{r^{2} / 4 k t}^{\infty} d u \frac{e^{-u}}{u} \\
& \times\left\{2 \operatorname{erf}\left(\frac{z}{r} \sqrt{u}\right)+\operatorname{erf}\left(\frac{L-z}{r} \sqrt{u}\right)-\operatorname{erf}\left(\frac{L+z}{r} \sqrt{u}\right)\right\},
\end{aligned}
$$

and the solution to (2.6), (2.7), and (2.9) is

$$
v_{d}(r, z, t)=\frac{Q_{z}}{8 \pi \lambda} \int_{r^{2} / 4 k t}^{\infty} \frac{e^{-u}}{u}\left\{\operatorname{erf}\left(\frac{z+L}{r} \sqrt{u}\right)-\operatorname{erf}\left(\frac{z-L}{r} \sqrt{u}\right)\right\} d u,
$$

that we will present in a future work.

\subsection{The Problem for $v_{0}$}

The partial differential equation that $v_{0}(z, t)$ satisfies is

$$
\frac{\partial v_{0}}{\partial t}=k \frac{\partial^{2} v_{0}}{\partial z^{2}}
$$

subject to the initial condition

$$
v_{0}(z, 0)=T_{0}+k_{\text {geo }} z, \quad z \geq 0,
$$

and to a Dirichlet boundary condition,

$$
v_{0}(0, t)=0, \quad t \geq 0
$$

or to a Neumann homogeneous boundary condition

$$
\partial_{z} v_{0}(0, t)=0, \quad t \geq 0
$$

The solution to (2.12), (2.13), and (2.14) is [5, Equation 2.4.13],

$$
v_{0}(z, t)=T_{0} \operatorname{erf}\left(\frac{z}{2 \sqrt{k t}}\right)+k_{\text {geo }} z
$$

and the solution to (2.12), (2.13), and (2.15) is [5, Equation 2.9.7]

$$
v_{0}(z, t)=T_{0}+k_{\text {geo }}\left\{z \operatorname{erf}\left(\frac{z}{2 \sqrt{k t}}\right)+\frac{2 \sqrt{k t}}{\sqrt{\pi}} \exp \left(-\frac{z^{2}}{4 k t}\right)\right\}
$$


where the error function is defined as

$$
\operatorname{erf}(z):=\frac{2}{\sqrt{\pi}} \int_{0}^{z} e^{-u^{2}} d u
$$

\subsection{The Problem for $v_{S}$}

The partial differential equation that $v_{s}(z, t)$ satisfies is

$$
\frac{\partial v_{s}}{\partial t}=k \frac{\partial^{2} v_{s}}{\partial z^{2}}
$$

subject to an homogeneous initial condition

$$
v_{s}(z, 0)=0, \quad z \geq 0,
$$

and to the time-dependent Dirichlet boundary condition

$$
v_{S}(0, t)=\psi(t), \quad t \geq 0,
$$

or to the time-dependent Neumann boundary condition

$$
-\lambda \partial_{z} v_{s}(0, t)=\psi(t), \quad t \geq 0
$$

The solution to (2.19), (2.20) and (2.21) is [5, Equation 2.5.1.]

$$
v_{S}(z, t)=\frac{2}{\sqrt{\pi}} \int_{z / 2 \sqrt{k t}}^{\infty} \psi\left(t-\frac{z^{2}}{4 k u^{2}}\right) e^{-u^{2}} d u
$$

and the solution to (2.19), (2.20) and (2.22) is [5, Equation 2.9.9]

$$
v_{s}(z, t)=\frac{\sqrt{k}}{\lambda \sqrt{\pi}} \int_{0}^{t} \frac{\psi(t-u)}{\sqrt{u}} e^{-z^{2} / 4 k u} d u
$$

\section{Harmonic Analysis of the Boundary Condition}

We may consider that on the ground surface we have a periodic temperature or heat flux oscillation, due to daily or annual cycles. Therefore, $\psi(t)$ is a periodic function of a certain period $\tau$, continuous and bounded, that we may expand in Fourier series

$$
\psi(t)=\frac{a_{0}}{2}+\sum_{n=1}^{\infty} a_{n} \cos (n \omega t)+b_{n} \sin (n \omega t)
$$


where the frequency is $\omega=2 \pi / \tau$, and the coefficients $a_{n}$ and $b_{n}$ are given by

$$
\begin{aligned}
& a_{0}=\frac{2}{\tau} \int_{-\tau / 2}^{\tau / 2} \psi(u) d u, \\
& a_{n}=\frac{2}{\tau} \int_{-\tau / 2}^{\tau / 2} \psi(u) \cos (n \omega u) d u \\
& b_{n}=\frac{2}{\tau} \int_{-\tau / 2}^{\tau / 2} \psi(u) \sin (n \omega u) d u,
\end{aligned}
$$

where $n \in \mathbb{N}$.

\subsection{Dirichlet Boundary Condition}

Substituting (3.1) in (2.23), we obtain

$$
\begin{aligned}
v_{s}(z, t)= & \frac{a_{0}}{2} \operatorname{erfc}\left(\frac{z}{2 \sqrt{k t}}\right) \\
& +\frac{2}{\sqrt{\pi}} \sum_{n=1}^{\infty} a_{n} \int_{z / 2 \sqrt{k t}}^{\infty} \cos \left(n \omega t-\frac{n \omega z^{2}}{4 k u^{2}}\right) e^{-u^{2}} d u \\
& +b_{n} \int_{z / 2 \sqrt{k t}}^{\infty} \sin \left(n \omega t-\frac{n \omega z^{2}}{4 k u^{2}}\right) e^{-u^{2}} d u,
\end{aligned}
$$

where $\operatorname{erfc}(z)=1-\operatorname{erf}(z)$ is the complementary error function. The integrals given in (3.3) for $t \geq 0$ may be rewritten as

$$
\begin{aligned}
& \int_{z / 2 \sqrt{k t}}^{\infty} \cos \left(n \omega t-\frac{n \omega z^{2}}{4 k u^{2}}\right) e^{-u^{2}} d u=\operatorname{Re}\left[e^{i n \omega t} I_{D, n}(z, t)\right], \\
& \int_{z / 2 \sqrt{k t}}^{\infty} \sin \left(n \omega t-\frac{n \omega z^{2}}{4 k u^{2}}\right) e^{-u^{2}} d u=\operatorname{Im}\left[e^{i n \omega t} I_{D, n}(z, t)\right],
\end{aligned}
$$

where we have defined

$$
I_{D, n}(z, t):=\int_{z / 2 \sqrt{k t}}^{\infty} \exp \left(-\frac{i n \omega z^{2}}{4 k u^{2}}-u^{2}\right) d u
$$

In order to calculate (3.5), let us apply the integral [6, Equation 7.4.33]

$$
\int \exp \left(-a x^{2}-\frac{b}{x^{2}}\right) d x=\frac{\sqrt{\pi}}{4 \sqrt{a}}\left[e^{2 \sqrt{a b}} \operatorname{erf}\left(\sqrt{a} x+\frac{\sqrt{b}}{x}\right)+e^{-2 \sqrt{a b}} \operatorname{erf}\left(\sqrt{a} x-\frac{\sqrt{b}}{x}\right)\right]+\text { const, }
$$


taking $a=1, b=i n \omega z^{2} / 4 k$, and $z \geq 0$, so that

$$
\begin{aligned}
I_{D, n}(z, t)=\frac{\sqrt{\pi}}{4}[ & \exp \left(\frac{z \sqrt{i n \omega}}{\sqrt{k}}\right) \operatorname{erfc}\left(\frac{z}{2 \sqrt{k t}}+\sqrt{i n \omega t}\right) \\
& \left.+\exp \left(\frac{-z \sqrt{i n \omega}}{\sqrt{k}}\right) \operatorname{erfc}\left(\frac{z}{2 \sqrt{k t}}-\sqrt{i n \omega t}\right)\right] .
\end{aligned}
$$

Finally, substituting (3.7) in (3.4), we obtain for $z, t \geq 0$,

$$
\begin{aligned}
& \int_{z / 2 \sqrt{k t}}^{\infty} \cos \left(n \omega t-\frac{n \omega z^{2}}{4 k u^{2}}\right) e^{-u^{2}} d u=\frac{\sqrt{\pi}}{4} \operatorname{Re}\left\{e^{i n \omega t}\left[A_{n}^{+}(z, t)+A_{n}^{-}(z, t)\right]\right\}, \\
& \int_{z / 2 \sqrt{k t}}^{\infty} \sin \left(n \omega t-\frac{n \omega z^{2}}{4 k u^{2}}\right) e^{-u^{2}} d u=\frac{\sqrt{\pi}}{4} \operatorname{Im}\left\{e^{i n \omega t}\left[A_{n}^{+}(z, t)+A_{n}^{-}(z, t)\right]\right\},
\end{aligned}
$$

where we have defined

$$
A_{n}^{ \pm}(z, t):=\exp \left( \pm \frac{z \sqrt{i n \omega}}{\sqrt{k}}\right) \operatorname{erfc}\left(\frac{z}{2 \sqrt{k t}} \pm \sqrt{i n \omega t}\right)
$$

As far as we know, the integrals given in (3.8) are not reported in the literature.

\subsubsection{Neumann Boundary Condition}

Substituting (3.1) in (2.24), we obtain

$$
\begin{aligned}
v_{S}(z, t)= & \frac{a_{0} \sqrt{k}}{2 \lambda \sqrt{\pi}} \int_{0}^{t} \frac{e^{-z^{2} / 4 k u}}{\sqrt{u}} d u \\
& +\frac{\sqrt{k}}{\lambda \sqrt{\pi}} \sum_{n=1}^{\infty} a_{n} \int_{0}^{t} \frac{\cos [n \omega(t-u)]}{\sqrt{u}} e^{-z^{2} / 4 k u} d u \\
& +b_{n} \int_{0}^{t} \frac{\sin [n \omega(t-u)]}{\sqrt{u}} e^{-z^{2} / 4 k u} d u
\end{aligned}
$$

Integrating by parts and performing the substitution $\zeta=z / 2 \sqrt{k u}$, considering $z, t \geq 0$, we may calculate the integral given in (3.10)

$$
\int_{0}^{t} \frac{e^{-z^{2} / 4 k u}}{\sqrt{u}} d u=2 \sqrt{t} \exp \left(\frac{-z^{2}}{4 k t}\right)-\frac{\sqrt{\pi} z}{\sqrt{k}} \operatorname{erfc}\left(\frac{z}{2 \sqrt{k t}}\right) .
$$


In order to calculate the integrals given in (3.11) and (3.12), let us perform the substitution $u=v^{2}$, considering $t \geq 0$, so that

$$
\begin{aligned}
\int_{0}^{t} \frac{\cos [n \omega(t-u)]}{\sqrt{u}} e^{-z^{2} / 4 k u} d u & =2 \int_{0}^{\sqrt{t}} \cos \left[n \omega\left(t-v^{2}\right)\right] e^{-z^{2} / 4 k v^{2}} d v \\
& =2 \operatorname{Re}\left[e^{i n \omega t} I_{N, n}(z, t)\right]
\end{aligned}
$$

and similarly

$$
\int_{0}^{t} \frac{\sin [n \omega(t-u)]}{\sqrt{u}} e^{-z^{2} / 4 k u} d u=2 \operatorname{Im}\left[e^{i n \omega t} I_{N, n}(z, t)\right]
$$

where we have defined

$$
I_{N, n}(z, t):=\int_{0}^{\sqrt{t}} \exp \left(-i n \omega v^{2}-\frac{z^{2}}{4 k v^{2}}\right) d v
$$

Taking $a=i n \omega, b=z^{2} / 4 k$, and $z \geq 0$ in (3.6), we may rewrite (3.16) as

$$
\begin{aligned}
I_{N, n}(z, t)=\frac{\sqrt{\pi}}{4 \sqrt{i n \omega}} & {\left[\exp \left(\frac{-z \sqrt{i n \omega}}{\sqrt{k}}\right) \operatorname{erfc}\left(\frac{z}{2 \sqrt{k t}}-\sqrt{i n \omega t}\right)\right.} \\
& \left.-\exp \left(\frac{z \sqrt{i n \omega}}{\sqrt{k}}\right) \operatorname{erfc}\left(\frac{z}{2 \sqrt{k t}}+\sqrt{i n \omega t}\right)\right] .
\end{aligned}
$$

Finally, substituting (3.17) in (3.14) and (3.15), for $z, t \geq 0$, we obtain

$$
\begin{aligned}
& \int_{0}^{t} \frac{\cos [n \omega(t-u)]}{\sqrt{u}} e^{-z^{2} / 4 k u} d u=\frac{\sqrt{\pi}}{2} \operatorname{Re}\left\{\frac{e^{i n \omega t}}{\sqrt{i n \omega}}\left[A_{n}^{-}(z, t)-A_{n}^{+}(z, t)\right]\right\} \\
& \int_{0}^{t} \frac{\sin [n \omega(t-u)]}{\sqrt{u}} e^{-z^{2} / 4 k u} d u=\frac{\sqrt{\pi}}{2} \operatorname{Im}\left\{\frac{e^{i n \omega t}}{\sqrt{i n \omega}}\left[A_{n}^{-}(z, t)-A_{n}^{+}(z, t)\right]\right\} .
\end{aligned}
$$

As far as we know, the integrals given in (3.18) are not reported in the literature.

\section{Particular Cases}

As a consistency check, let us verify that in certain particular cases the new integrals given in (3.8) and (3.18) are reduced to integrals reported in the literature. These particular cases have to do with the quasistationary regime and the solution on the ground surface. 


\subsection{Quasistationary Regime}

Since there is a periodic oscillation on the ground surface, the surroundings of the borehole heat exchanger never reach a stationary regime. However, according to [5, Section 2.6], we may define a quasistationary regime in which the ground temperature field is stabilized periodically. In this quasistationary regime, (3.8) becomes

$$
\begin{aligned}
& \int_{0}^{\infty} \cos \left(n \omega t-\frac{n \omega z^{2}}{4 k u^{2}}\right) e^{-u^{2}} d u=\frac{\sqrt{\pi}}{4} \operatorname{Re}\left\{e^{i n \omega t} A_{D, n}(z)\right\}, \\
& \int_{0}^{\infty} \sin \left(n \omega t-\frac{n \omega z^{2}}{4 k u^{2}}\right) e^{-u^{2}} d u=\frac{\sqrt{\pi}}{4} \operatorname{Im}\left\{e^{i n \omega t} A_{D, n}(z)\right\},
\end{aligned}
$$

where we have defined

$$
A_{D, n}(z):=\lim _{t \rightarrow \infty}\left[A_{n}^{+}(z, t)+A_{n}^{-}(z, t)\right]
$$

Similarly, (3.18) becomes

$$
\begin{aligned}
& \int_{0}^{\infty} \frac{\cos [n \omega(t-u)]}{\sqrt{u}} e^{-z^{2} / 4 k u} d u=\frac{\sqrt{\pi}}{2} \operatorname{Re}\left\{\frac{e^{i n \omega t}}{\sqrt{i n \omega}} A_{N, n}(z)\right\} \\
& \int_{0}^{\infty} \frac{\sin [n \omega(t-u)]}{\sqrt{u}} e^{-z^{2} / 4 k u} d u=\frac{\sqrt{\pi}}{2} \operatorname{Im}\left\{\frac{e^{i n \omega t}}{\sqrt{i n \omega}} A_{N, n}(z)\right\},
\end{aligned}
$$

where we have defined

$$
A_{N, n}(z):=\lim _{t \rightarrow \infty}\left[A_{n}^{-}(z, t)-A_{n}^{+}(z, t)\right]
$$

\subsubsection{Dirichlet Case}

Taking into account that $\operatorname{erfc}(\infty)=0$ and $\operatorname{erfc}(-\infty)=2$, we may calculate the limit given in (4.3), so that (4.1) becomes

$$
\begin{aligned}
\int_{0}^{\infty} \cos \left(n \omega t-\frac{n \omega z^{2}}{4 k u^{2}}\right) e^{-u^{2}} d u & =\frac{\sqrt{\pi}}{2} \operatorname{Re}\left\{e^{i n \omega t} \exp \left(\frac{-z \sqrt{i n \omega}}{\sqrt{k}}\right)\right\} \\
& =\frac{\sqrt{\pi}}{2} e^{-z \sqrt{n \omega / 2 k}} \operatorname{Re}\left\{e^{i(n \omega t-z \sqrt{n \omega / 2 k})}\right\},
\end{aligned}
$$

where we have substitute $\sqrt{i}=(1+i) / \sqrt{2}$. Thus, for $z, t \geq 0$,

$$
\int_{0}^{\infty} \cos \left(n \omega t-\frac{n \omega z^{2}}{4 k u^{2}}\right) e^{-u^{2}} d u=\frac{\sqrt{\pi}}{2} e^{-z \sqrt{n \omega / 2 k}} \cos \left(n \omega t-\frac{z \sqrt{n \omega}}{\sqrt{2 k}}\right) .
$$


Similarly, we may obtain

$$
\int_{0}^{\infty} \sin \left(n \omega t-\frac{n \omega z^{2}}{4 k u^{2}}\right) e^{-u^{2}} d u=\frac{\sqrt{\pi}}{2} e^{-z \sqrt{n \omega / 2 k}} \sin \left(n \omega t-\frac{z \sqrt{n \omega}}{\sqrt{2 k}}\right) .
$$

Notice that (4.7) and (4.8) are sinusoidal in $t$, with an amplitude exponentially decreasing in depth $z$. In the literature, we may find [7, Equation 3.928.1-2].

$$
\begin{aligned}
\int_{0}^{\infty} e^{-p^{2} x^{2}-q^{2} / x^{2}} \sin \left(a^{2} x^{2}+\frac{b^{2}}{x^{2}}\right) d x= & \frac{\sqrt{\pi}}{2 r} e^{-2 r s \cos (A+B)} \\
& \times \sin (A+2 r s \sin (A+B)), \\
\int_{0}^{\infty} e^{-p^{2} x^{2}-q^{2} / x^{2}} \cos \left(a^{2} x^{2}+\frac{b^{2}}{x^{2}}\right) d x= & \frac{\sqrt{\pi}}{2 r} e^{-2 r s \cos (A+B)} \\
& \times \cos (A+2 r s \sin (A+B)),
\end{aligned}
$$

where $a^{2}+p^{2}>0$ and

$$
\begin{gathered}
r=\sqrt[4]{a^{4}+p^{4}}, \quad s=\sqrt[4]{b^{4}+q^{4}} \\
A=\frac{1}{2} \tan ^{-1}\left(\frac{a^{2}}{p^{2}}\right), \quad B=\frac{1}{2} \tan ^{-1}\left(\frac{b^{2}}{q^{2}}\right),
\end{gathered}
$$

thus, taking $p=1, q=0$, and $a=0$,

$$
\begin{aligned}
& \int_{0}^{\infty} e^{-x^{2}} \sin \left(\frac{b^{2}}{x^{2}}\right) d x=\frac{\sqrt{\pi}}{2} e^{-\sqrt{2} b} \sin (\sqrt{2} b) \\
& \int_{0}^{\infty} e^{-x^{2}} \cos \left(\frac{b^{2}}{x^{2}}\right) d x=\frac{\sqrt{\pi}}{2} e^{-\sqrt{2} b} \cos (\sqrt{2} b) .
\end{aligned}
$$

Therefore, rewriting the left side of (4.7) as

$$
\begin{aligned}
\int_{0}^{\infty} \cos \left(n \omega t-\frac{n \omega z^{2}}{4 k u^{2}}\right) e^{-u^{2}} d u= & \cos (n \omega t) \int_{0}^{\infty} \cos \left(\frac{n \omega z^{2}}{4 k u^{2}}\right) e^{-u^{2}} d u \\
& +\sin (n \omega t) \int_{0}^{\infty} \sin \left(\frac{n \omega z^{2}}{4 k u^{2}}\right) e^{-u^{2}} d u
\end{aligned}
$$

and applying (4.11), taking $b=\sqrt{n \omega} z / 2 \sqrt{k},(z \geq 0)$, we eventually get the same result as (4.7). Similarly, we may check the result given in (4.8). 


\subsubsection{Neumann Case}

In a similar way as in the previous subsection, we may calculate (4.4) for $z, t \geq 0$, arriving at

$$
\begin{aligned}
& \int_{0}^{\infty} \frac{\cos [n \omega(t-u)]}{\sqrt{u}} e^{-z^{2} / 4 k u} d u=\frac{\sqrt{\pi} e^{-z \sqrt{n \omega / 2 k}}}{\sqrt{n \omega}} \cos \left(n \omega t-\frac{z \sqrt{n \omega}}{\sqrt{2 k}}-\frac{\pi}{4}\right), \\
& \int_{0}^{\infty} \frac{\sin [n \omega(t-u)]}{\sqrt{u}} e^{-z^{2} / 4 k u} d u=\frac{\sqrt{\pi} e^{-z \sqrt{n \omega / 2 k}}}{\sqrt{n \omega}} \sin \left(n \omega t-\frac{z \sqrt{n \omega}}{\sqrt{2 k}}-\frac{\pi}{4}\right) .
\end{aligned}
$$

Once again, (4.13) and (4.7) are sinusoidal in $t$, with an amplitude exponentially decreasing in depth $z$. In the literature, we may find [7, Equation 3.957.1-2]

$$
\begin{aligned}
& \int_{0}^{\infty} x^{\mu-1} e^{-\beta^{2} / 4 x} \cos a x d x=\left(\frac{\beta}{2 \sqrt{a}}\right)^{\mu}\left[e^{-i \pi \mu / 4} K_{\mu}\left(\beta e^{i \pi / 4} \sqrt{a}\right)+e^{i \pi \mu / 4} K_{\mu}\left(\beta e^{-i \pi / 4} \sqrt{a}\right)\right], \\
& \int_{0}^{\infty} x^{\mu-1} e^{-\beta^{2} / 4 x} \sin a x d x=i\left(\frac{\beta}{2 \sqrt{a}}\right)^{\mu}\left[e^{-i \pi \mu / 4} K_{\mu}\left(\beta e^{i \pi / 4} \sqrt{a}\right)-e^{i \pi \mu / 4} K_{\mu}\left(\beta e^{-i \pi / 4} \sqrt{a}\right)\right],
\end{aligned}
$$

where $\operatorname{Re} \beta>0, \operatorname{Re} \mu<1$ and $a>0$. Therefore, taking in (4.15), $\mu=1 / 2, a=n \omega, \beta=z / \sqrt{k}$, $(z \geq 0)$, and knowing that the Macdonald function of order $1 / 2$ is [8, Equation 5.5.5],

$$
K_{1 / 2}(z)=\sqrt{\frac{\pi}{2 z}} e^{-z}
$$

we eventually get the same results as (4.13) and (4.14).

\subsection{Solution on the Ground Surface}

Notice that in the special case $z=0,(3.8)$ becomes trivial,

$$
\begin{aligned}
& \int_{0}^{\infty} \cos (n \omega t) e^{-u^{2}} d u=\frac{\sqrt{\pi}}{2} \cos (n \omega t), \\
& \int_{0}^{\infty} \sin (n \omega t) e^{-u^{2}} d u=\frac{\sqrt{\pi}}{2} \sin (n \omega t),
\end{aligned}
$$

while (3.18) are reduced to

$$
\begin{aligned}
& \int_{0}^{t} \frac{\cos [n \omega(t-u)]}{\sqrt{u}} d u=\sqrt{\pi} \operatorname{Re}\left\{\frac{e^{i n \omega t}}{\sqrt{i n \omega}} \operatorname{erf}(\sqrt{i n \omega t})\right\}, \\
& \int_{0}^{t} \frac{\sin [n \omega(t-u)]}{\sqrt{u}} d u=\sqrt{\pi} \operatorname{Im}\left\{\frac{e^{i n \omega t}}{\sqrt{i n \omega}} \operatorname{erf}(\sqrt{i n \omega t})\right\} .
\end{aligned}
$$


Since [8, Equation 2.4.1]

$$
\frac{\operatorname{erf}(\sqrt{i} x)}{\sqrt{i}}=\sqrt{2}\left[C\left(\sqrt{\frac{2}{\pi}} x\right)-i S\left(\sqrt{\frac{2}{\pi}} x\right)\right], \quad x \in \mathbb{R}
$$

where

$$
\begin{aligned}
& C(z):=\int_{0}^{z} \cos \frac{\pi t^{2}}{2} d t \\
& S(z):=\int_{0}^{z} \sin \frac{\pi t^{2}}{2} d t
\end{aligned}
$$

are the Fresnel integrals; then (4.18) may be expressed as

$$
\begin{aligned}
& \int_{0}^{t} \frac{\cos [n \omega(t-u)]}{\sqrt{u}} d u=\sqrt{\frac{2 \pi}{n \omega}}\left[\cos (n \omega t) C\left(\sqrt{\frac{2 n \omega t}{\pi}}\right)+\sin (n \omega t) S\left(\sqrt{\frac{2 n \omega t}{\pi}}\right)\right], \\
& \int_{0}^{t} \frac{\sin [n \omega(t-u)]}{\sqrt{u}} d u=\sqrt{\frac{2 \pi}{n \omega}}\left[\sin (n \omega t) C\left(\sqrt{\frac{2 n \omega t}{\pi}}\right)-\cos (n \omega t) S\left(\sqrt{\frac{2 n \omega t}{\pi}}\right)\right] .
\end{aligned}
$$

The results given in (4.21), agrees with [7, Equation 2.653.1-2]

$$
\begin{aligned}
& \int \frac{\sin x}{\sqrt{x}} d x=\sqrt{2 \pi} S(\sqrt{x}), \\
& \int \frac{\cos x}{\sqrt{x}} d x=\sqrt{2 \pi} C(\sqrt{x}) .
\end{aligned}
$$

\section{Acknowledgments}

The authors wish to thank the financial support received from Generalitat Valenciana under Grant no. GVA 3012/2009 and from Universidad Politécnica de Valencia under Grant no. PAID-06-09. This work has been partially supported by the Structural Funds of the European Regional Development Fund (ERDF).

\section{References}

[1] J. F. Urchueguía, M. Zacarés, J. M. Corberán, A. Montero, J. Martos, and H. Witte, “Comparison between the energy performance of a ground coupled water to water heat pump system and an air to water heat pump system for heating and cooling in typical conditions of the European Mediterranean coast," Energy Conversion and Management, vol. 49, no. 10, pp. 2917-2923, 2008.

[2] J. W. Lund, "Ground source (geothermal) heat pumps," in Course on Heating with Geothermal Energy: Conventional and New Schemes. World Geothermal Congress, P. J. Lineau, Ed., pp. 1-21, Kazuno, Japan, 2000, Short Courses. 
[3] T. V. Bandos, A. Montero, E. Fernández et al., "Finite line-source model for borehole heat exchangers: effect of vertical temperature variations," Geothermics, vol. 38, no. 2, pp. 263-270, 2009.

[4] P. Eskilson, Thermal analysis of heat extraction boreholes, Ph.D. thesis, Department of Mathematical Physics, University of Lund, Lund, Sweden, 1987.

[5] H. S. Carslaw and J. C. Jaeger, Conduction of Heat in Solids, Oxford Science Publications, The Clarendon Press, Oxford University Press, New York, NY, USA, 2nd edition, 1988.

[6] M. Abramowitz and I. A. Stegun, Handbook of Mathematical Functions, Dover Publications Paperback, New York, NY, USA, 1972.

[7] I. S. Gradshteyn and I. M. Ryzhik, Table of Integrals, Series, and Products, Elsevier/Academic Press, Amsterdam, Netherlands, 7th edition, 2007.

[8] N. N. Lebedev, Special Functions and Their Applications, Dover, New York, NY, USA, 1972. 


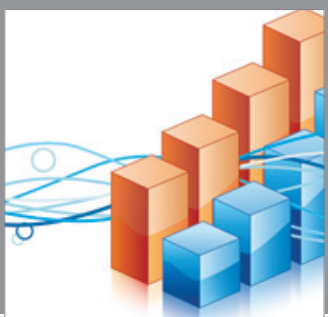

Advances in

Operations Research

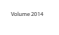

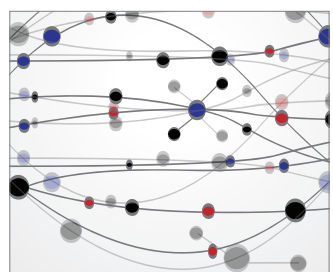

\section{The Scientific} World Journal
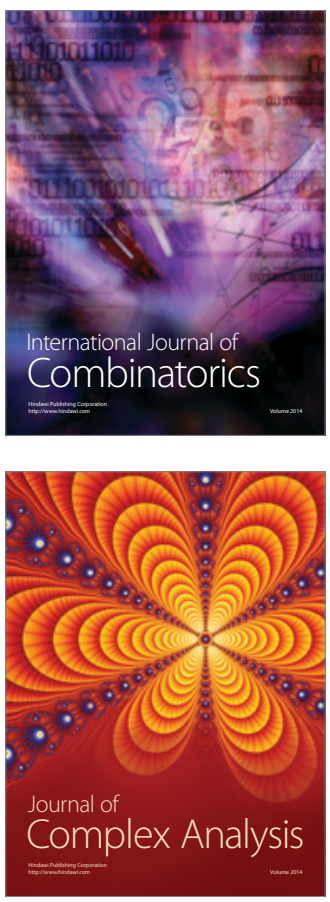

International Journal of

Mathematics and

Mathematical

Sciences
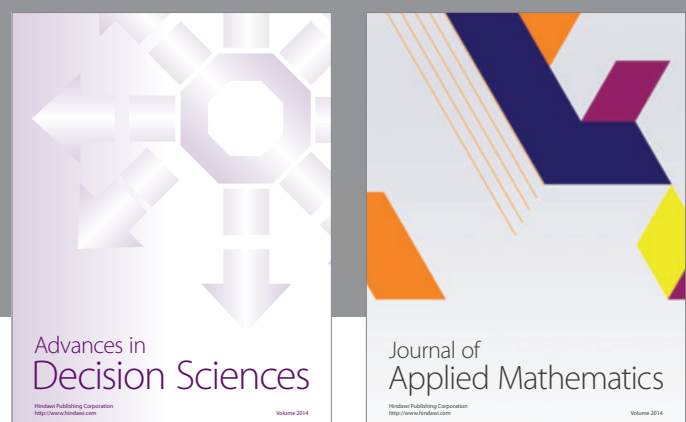

Journal of

Applied Mathematics
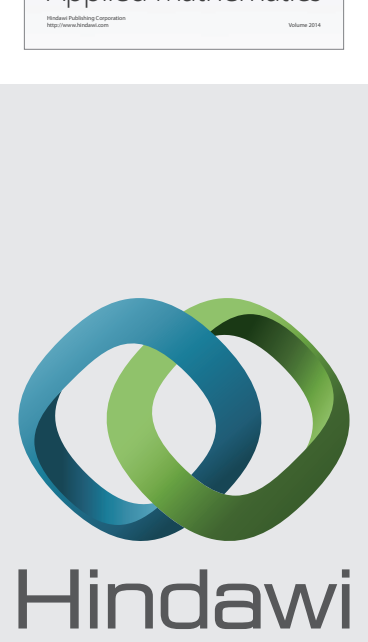

Submit your manuscripts at http://www.hindawi.com
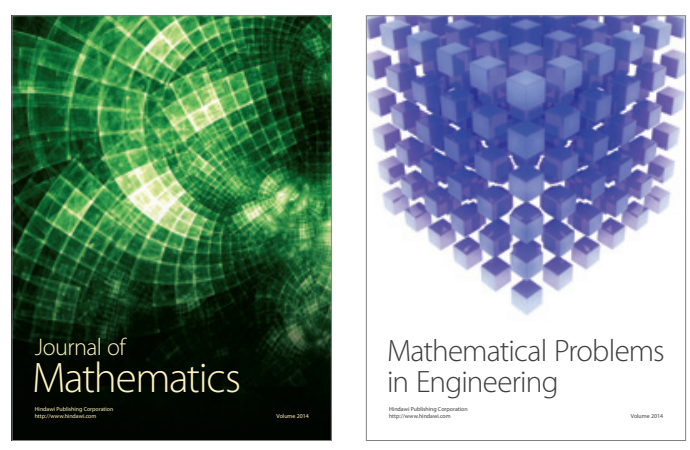

Mathematical Problems in Engineering
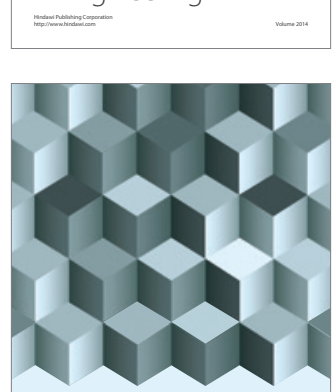

Journal of

Function Spaces
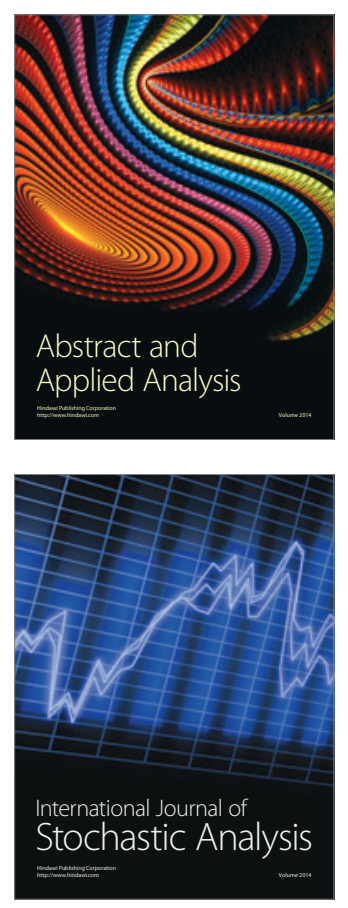

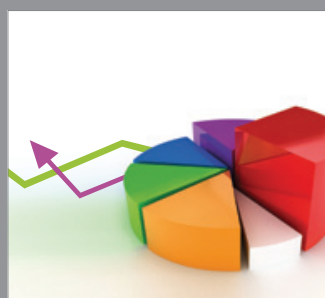

ournal of

Probability and Statistics

Promensencen
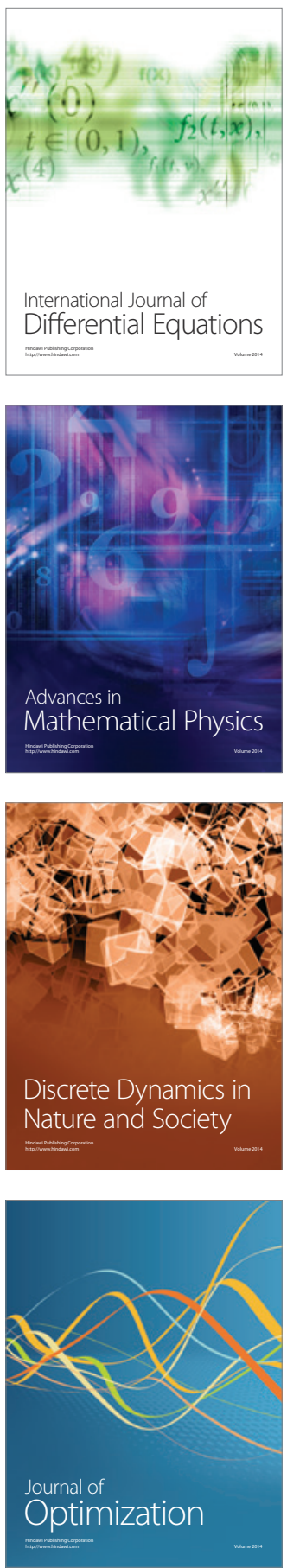